\title{
Mirada aproximativa a la construcción de la noticia sobre lo peruano en la prensa diaria de cobertura nacional ("El Mercurio", 2010). Un Análisis Crítico del Discurso (ACD) ${ }^{1}$
}

\author{
Approach to the construction of the news in the daily \\ nationwide press ("El Mercurio", 2010) involving Peruvian \\ issues. A Critical Discourse Analysis (CDA). ${ }^{2}$
}

\section{Rodrigo Browne Sartori}

Instituto de Comunicación Social, Universidad Austral de Chile rodrigobrowne@uach.cl

\section{Ricardo Baessolo Stiven}

Núcleo de Comunicación, Universidad Austral de Chile ricardobaessolo@gmail.com

\begin{abstract}
Resumen
Esta investigación pretende develar los mecanismos por los cuales la prensa nacional genera y representa discursos culturales provenientes de la convivencia bilateral entre peruanos y chilenos.

A través de un Análisis Crítico del Discurso (ACD) de noticias de principios de 2010 en el periódico "El Mercurio" -perteneciente a uno de los grupos periodísticos con mayor tiraje en Chile (El Mercurio S.A.P.)- se propone una mirada aproximativa a aquellos procesos de construcción social de la realidad utilizados para representar los discursos de la diferencia que se traducen en discriminación, generalizaciones y xenofobia sobre, principalmente, lo peruano, marcando significativas distancias con nosotros: los chilenos.
\end{abstract}

Palabras clave: Construcción social de la realidad, Análisis Crítico del Discurso, interculturalidad, periodismo, discurso de la diferencia.

\footnotetext{
${ }^{1}$ Este artículo forma parte del proyecto Fondecyt n 1100264 "Periodismo intercultural: Construcción de la noticia a través de un análisis crítico y complejo del discurso en la prensa diaria de cobertura nacional de Chile y Perú. El caso de 'El Mercurio' y 'La Cuarta' y 'El Comercio’ y 'Ajá’”.

${ }^{2}$ This work is part of the Fondecyt project n ${ }^{\circ} 1107064$ "Intercultural Journalism: Social Construction of the News through a Critical and Complex Discourse Analysis in the Chilean and Peruvian Nationwide Daily Press. The case of 'El Mercurio' y 'La Cuarta' $y^{2}$ 'El Comercio' y 'Ajá”'.
} 


\begin{abstract}
This research intends to unveil the mechanisms through which the nationwide press generates and represents the cultural discourses coming from the bilateral coexistence between Peruvians and Chileans.

Carrying out a Critical Discourse Analysis (CDA) of the news of the beginnings of 2010, extracted from "El Mercurio" -which belongs to the consortium that controls the nationwide informative issuing in Chile (El Mercurio S.A.P.)- this paper proposes an approach to the processes of social construction of the reality used to represent the discourses of difference which have resulted into discrimination, generalisations and xenophobia concerning, mainly, the Peruvian issues, marking significant gaps with us: the Chileans.
\end{abstract}

Key words: Social construction of reality, Critical Discourse Analysis (CDA), intercultural communication and journalism, discourse of the difference.

\title{
1. Constructivismo e identidad cultural
}

Desde una visión constructivista, la identidad puede concebirse como una construcción cultural generada por la socialización e interacción social, lo que implica que no es inherente al hombre, sino que se construye, y lo hace por comparación y diferenciación con el otro; para adquirir una identidad es necesario conocerse a través de los otros. Por esto, Rodrigo Alsina (1999) diferencia dos niveles de identidad: la paradigmática y la pragmática. La identidad paradigmática implica que las personas cuentan con una serie de modelos históricamente establecidos y socialmente connotados que se renuevan constantemente. El nivel pragmático de la construcción identitaria implica que ésta nace de la interacción con los otros, adquiriendo su forma en las relaciones intersubjetivas entre mismos y otros.

De igual manera, Grimson (2001) afirma que la primera categoría de la identidad es su carácter Relacional, es decir, al momento de definir un nosotros, automáticamente se define un otro. Esta categoría es fundamental para entender la correlación entre culturas bajo parámetros de subordinación y coordinación, lo que podría implicar la existencia de categorías jerárquicas al establecer la otredad cultural.

En esta misma línea, el estudio de Peter Berger y Thomas Luckmann (1972) se opone a la Teoría del Conocimiento de Max Scheler de los años 20, que centraba su interés teórico en cuestiones epistemológicas y aplicaba sus trabajos a la historia de las ideas. Es decir, las teorizaciones no bastaban para Berger y Luckmann, ya que creían que éstas eran sólo una parte del conocimiento. Por lo mismo, postulan que el objeto de estudio deben ser los integrantes de una sociedad, ya que los individuos tienen ciertas certezas sobre el mundo que los rodea, lo que conforma sus realidades, entendiendo realidad como la "cualidad 
propia de los fenómenos que reconocemos como independientes de nuestra propia volición" (1972: 13) y conocimiento como "la certidumbre de que los fenómenos son reales y de que poseen características específicas" (1972: 13). Ambas definiciones se realizan en un contexto sociológico específico, destinado a analizar la construcción de realidades desde la práctica social, y no se deben aplicar al campo filosófico que pretende, de alguna manera, encontrar el origen a preguntas existencialistas muy por encima de la cuestión social.

Esta característica pasiva que Berger y Luckmann le otorgan al hombre común y corriente sobre la percepción dada de la realidad y el conocimiento es trascendental para comenzar a distinguir el problema emergente de que un factor externo pueda influir en la sociedad dando percepciones que las masas absorberán sin cuestionarse, es decir, imponer construcciones sobre la realidad. Hablar de este factor externo con poder sobre la sociedad pasiva no es un eufemismo para referirse exclusivamente a los medios de comunicación objeto de análisis en esta investigación- ya que existen otros más poderosos, incluso capaces de influir fuertemente en los medios: los que influyen a los que influyen. Es así que podemos asociar a los medios de comunicación con tendencias políticas muy bien definidas y acotadas que, directa o indirectamente, mantienen una permanente tutela sobre estos individuos pasivos.

El interés sociológico sobre realidad y conocimiento se basa en el principio de la relatividad social, que implica que el contexto social de un individuo hará que sus percepciones sobre lo real y el conocimiento sean distintas a los de otro individuo con un contexto social diferente. En el caso de los medios de comunicación, que suponen tener un target específico, el carácter de la relatividad social debe contenerse, exclusivamente, en el corpus de la noticia, pues de ninguna otra manera podrían llegar a asociar a un conjunto de individuos diferentes dentro del mismo grupo objetivo. La noticia y su corpus son el medio y a la vez proceso por el cual un conocimiento llega a quedar establecido como realidad, "la vida cotidiana se presenta como una realidad interpretada por los hombres y que para ellos tiene el significado subjetivo de un mundo coherente" (Berger y Luckmann, 1972: 36), lo que da sustento a la idea de que es un mundo originado en los pensamientos y acciones de los individuos.

El sentido común involucra, necesariamente, ciertas interpretaciones pre y cuasicientíficas como, por ejemplo, la historia, por lo que para describir la realidad del sentido común es necesario referirse a ellas. "La conciencia es siempre intencional, siempre apunta o se dirige a objetos" (Berger y Luckmann, 1972: 38). Esta frase ayuda a comprender que la conciencia se mueve constantemente en diferentes esferas de la realidad, por lo general entre la conformada por el contacto con los objetos y los sentimientos o recuerdos de sensaciones. En otras palabras, los individuos tienen conciencia de que existen realidades múltiples dentro de sus mundos. 
La realidad de la vida cotidiana se presenta como la realidad por excelencia, o "suprema realidad" en palabras de Berger y Luckmann, pues es natural desenvolverse en ella con plena vigilia. Es una realidad que está al alcance corporal de los individuos, lo que permite manipularla y ejercer actividades en ella. En esta suprema realidad, la conciencia está dominada por un motivo pragmático, es decir, "mi atención a este mundo está determinada principalmente por lo que hago, lo que ya he hecho o lo que pienso hacer en él” (Berger y Luckmann, 1972: 40). Este punto es muy importante de analizar, ya que plantea que el interés que tiene un individuo con un tema particular está determinado por su cercanía física con él. Esto hace que los intereses más cercanos se presenten con una urgencia predominante frente a tópicos más lejanos, quizás a nivel de pasatiempo, pero que no cuentan con la misma jerarquía que aquella temática que influye directamente en el mundo pragmático del individuo. Lo interesante es que ya podemos justificar que las estrategias utilizadas por los medios de comunicación pretenden influenciar en las percepciones de la realidad de los individuos a través de los temas que más les afecten. $\mathrm{Si}$ bien los medios de comunicación masivos se han caracterizado por apuntar a una realidad más global que local, la manera de llevar los intereses cercanos a la práctica de los individuos está dada, desde un principio, en sus secciones, las cuales han sido impuestas y han permanecido en el tiempo con tanta fuerza que hoy en día a nadie le sorprende encontrarse con política, nacional, deportes, editorial, etc. al abrir el diario. El ejemplo de las secciones del diario es necesario para comprender cómo los medios de comunicación manipulan y guían la realidad encasillándola en temáticas que pueden llegar de mejor manera hacia los intereses de los individuos según sus actividades de mayor interés y urgencia.

Además, la vida cotidiana se presenta como una realidad ordenada, en el sentido de que los fenómenos que en ella aparecen son dispuestos con anterioridad en pautas que se imponen independientemente de las interpretaciones de cada quien. Este orden hace que la realidad de la vida cotidiana se presente como objetiva, sobre todo gracias al lenguaje utilizado en ella que, por un lado, nombra los objetos, situaciones y fenómenos para ordenar aquello que tiene significado para los individuos y, por otro, permite la formación de redes de contacto en las que cada cual se desenvuelve. En palabras de Berger y Luckmann (1972: 39), "el lenguaje marca las coordenadas de mi vida en la sociedad y llena esa vida de objetos significativos”.

\section{Comunicación intercultural y medios de comunicación}

Después de revisar, en general, cuestiones básicas de la construcción social de la realidad, podríamos advertir, a modo de resumen, que la complejidad que implica que un individuo 
pretenda comprender una realidad inabarcable facilita la generalización de características culturales distintas, bajo estereotipos reflejados en las barreras subjetivas de la experiencia de los mismos individuos. Individuos limitados por la realidad que transan su incapacidad de experimentar, cara a cara, el mundo que los rodea y sus relaciones interpersonales por la posibilidad de que un tercero se las resuma: los medios de comunicación.

Gracias a lo anteriormente planteado, podemos fijar el punto de partida de los problemas de la comunicación intercultural en el desconocimiento que una cultura tiene de otra. El problema radica en que siempre existirá un grado de desconocimiento, sobre todo si la globalización implica una saturación de información, por lo que este punto de partida debe ser entendido, también, como un punto de llegada. La generalización -llamada "sobregeneralización” por Miquel Rodrigo Alsina (1999: 64)- permite explicar, fácilmente, un mundo que no está a nuestro alcance, pues "nos permite una economía mental, ya que el estereotipo preconcebido facilita la explicación de la realidad. El fijarse atentamente y el intentar descubrir el sentido de las cosas se vuelve innecesario".

Es así como por costumbre, y con una naturalidad que a simple vista no desconoceríamos, tendemos a referirnos a las agrupaciones culturales distintas como los peruanos, los bolivianos, los mapuches, etc., sobre todo si reparamos en esos otros que cada vez más se ven reflejados en las páginas de los periódicos nacionales. Representación que, en este trabajo en particular y a modo de ejemplo, se detendrá en el cruce de los peruanos con y en el diario "El Mercurio".

Según Rodrigo Alsina, el desconocimiento sobre otra cultura permite que se establezca una primera relación basada en la comparación, donde un individuo, en base a su experiencia limitada, identifica características iguales, similares o diferentes con su propia cultura. Lo interesante de esto es que al utilizar un sistema comparativo para describir a otra cultura se construye la figura de el otro resaltando las diferencias, asumiendo que una cultura distinta es una cultura totalmente diferente, ya que el desconocimiento y la comprensión limitada de la realidad "solo puede, generalmente, proporcionar una visión diferenciadora" (1999: 65).

Como ya vimos, inmediatamente ante la conformación de un nosotros se construye un otro, lo que refuerza la idea de que existe un primer paso comparativo en la comunicación entre dos o más culturas. Pero, al convenir que este otro se caracteriza bajo tendencias diferenciadoras, resaltando lo distinto y no lo común, podemos comenzar a identificar los desarrollos de los conflictos interculturales desde la idea de que la diferenciación faculta una jerarquía valórica, donde lo distinto se percibe como malo o incorrecto y donde lo propio es lo bueno o correcto. Lo que Ximena Póo, a partir de la construcción de imaginarios sobre los otros y desde una mirada crítica al multiculturalismo, llama "los otros fronterizos", "estereotipos en donde el proyecto unificador de heterogeneidades muchas veces asume una agenda homogénea y hegemónica” (2009: 2). 
En este sentido, se podría decir que los medios de comunicación se guían por las características culturales de sus audiencias, por cuanto adaptan sus contenidos a un lenguaje determinado y a una visión sobre lo externo que concuerda con la manera en que los individuos (públicos o audiencias) realizan comparaciones diferenciadoras. Por ningún motivo esta investigación pretende validar este actuar, sobre todo tomando en cuenta que el pilar de esta demostración radica en la característica integradora de la comunicación, pero es interesante destacar que, por lo menos en este sentido, los medios son un reflejo de la cultura dominante, pues quiere decir que los medios tienen la capacidad de defender intereses que no sean propios. Los medios manejan acotados patrones culturales para consensuar sus interpretaciones hacia un público masivo que coincide con pertenecer a la cultura dominante, por lo que, con esto, deja de lado a todos los demás grupos que conforman la sociedad.

En consecuencia, los medios de comunicación, al reproducir y reforzar el discurso de las élites, no sólo terminan legitimando la supremacía de las culturas dominantes, sino que disminuyen y hasta invisibilizan al resto de agrupaciones no masivas. Lo importante es comprender que ambas consecuencias conforman un único esbozo de la realidad que es presentado por igual a los consumidores de información para guiar sus propias construcciones e imaginarios sobre lo social.

Francesc Barata explica, desde un enfoque interaccionista, que los medios de comunicación influyen en sus audiencias a través de la modelación del conocimiento, ya que "se les concibe como agentes modeladores del saber y reafirmadores del statu quo. Bajo estos planteamientos, el poder de los medios es decisivo en la construcción social de la realidad" (2006: 265). Este autor también reconoce la influencia del paradigma construccionista al destacar el papel de los medios no sólo en la creación de realidades marginales y marginadas, sino que en su influencia social y en "las formas de crear conocimientos sobre el otro estigmatizado y perseguido por las instituciones de control" (2006: 265).

La capacidad de los medios de comunicación de crear conocimientos, en este proceso de modelamiento del saber, ha sido estudiada, mayoritariamente, desde el campo de la sociología, donde nace la idea de que los medios masivos de comunicación realizan una criminalización de ciertos actos sociales, lo que nos recuerda la jerarquización valórica que realiza una cultura dominante para auto validarse e identificarse en tal proceso. Es así como se reconoce en los medios un proceso de etiquetamiento social, donde se definen ciertos actos y grupos de personas como amenazas al orden determinado utilizando estigmas y estereotipos. Por ejemplo, tomando un caso chileno, el año 2008 crece la preocupación por los temas de seguridad ciudadana, delincuencia y violencia gracias a que en el contexto social y político del país -reflejados en las principales encuestas de opinión- surgen estas temáticas como las prioridades del pueblo, demandando acciones inmediatas por parte del 
gobierno de turno.

A raíz de esto, los medios de comunicación comienzan a saturar el mercado informativo con reportajes sobre dicha temática. Pero, paralelamente, la prensa presenta, con un interés creciente, los incendios en cités del centro de Santiago donde los inmigrantes peruanos ilegales viven en condiciones precarias, por lo que se crea la relación o conexión entre inmigrantes y seguridad ciudadana como tópicos de actualidad. Es así como los medios tachan las condiciones de vida de los peruanos ilegales como infrahumanas, por lo que actúa el proceso de etiquetamiento social y se dispone, ante la audiencia, al pueblo peruano como un grupo peligroso para el bienestar de la sociedad. En este ámbito es importante reconocer que el etiquetamiento social que realizan los medios podría relacionarse con la práctica periodística, en el entendido de que el tratamiento de las fuentes busca validar las voces oficiales dando mayor credibilidad a las instituciones y a sus actores.

En conclusión, se puede decir que las prácticas periodísticas operan discursivamente. Rodrigo Alsina (2005) reconoce que el discurso periodístico opera en tres instancias de la realidad: el mundo real, el mundo de referencia y el mundo posible. El mundo real es donde suceden hechos y acontecimientos fácticos. El mundo de referencia corresponde a una imagen del mundo real, pero estructurada en un sistema de selección de acontecimientos; en el caso de la prensa podemos identificar el mundo de referencia en las secciones del periódico y en la importancia que se le da a cada noticia. Por último, el mundo posible corresponde a una imagen del mundo real, pero creada por la narración de un hecho que sigue la estructura del mundo de referencia.

El mundo real se rige por procesos de verificación, pues la representación de los sucesos busca métodos de comprobación para su validación. En el mundo de referencia es más importante que el discurso sea inteligible para que pueda llegar a la mayor cantidad de audiencias. En el mundo posible se aplica un formato y estilo al suceso que lo transforma en una noticia.

De lleno en el producto periodístico, Martínez Nicolás (1996) propone cuatro operaciones donde se distinguen los procedimientos utilizados por los medios para crear discursos: valoración, tematización, actorización y referencias. La valorización corresponde al proceso de selección y jerarquización de fenómenos sociales, donde se le da una importancia a los hechos que conforman la selección noticiosa. Lo interesante de esto es que la práctica constante de los medios de comunicación, al realizar este proceso de selección y jerarquización, da origen a la agenda temática de los medios, que consiste en el conjunto de temas que le ofrecen a sus audiencias. Así, la agenda temática se transforma en el primer signo de intencionalidad con la que los medios proponen una determinada lectura de la realidad. Además, la repetición de temas en la agenda de los medios permite que las audiencias generen opinión pública, ya que causan una preocupación social.

La tematización actúa cuando la práctica periodística aplica su discurso a determinados 
temas de la agenda. Cuando se organiza el discurso en torno a las temáticas recurrentes terminan por establecer referencias o puntos de vistas para decodificar los acontecimientos y para interpretar la realidad.

La actorización se refiere a las voces y autores presentes en el discurso periodístico, los que pueden aparecer en el relato o ser referidos por él. La producción periodística otorgará una dimensión pública a determinados actores, mientras que silenciará a otros; no todas las personas que se encuentran implicadas en un hecho calificarán como actores públicos. Por esto, el concepto de actorización tiene, en primer lugar, una dimensión que otorga cierto grado de participación de los actores en la información y, en segundo lugar, una dimensión que otorga sus grados de visibilidad.

La referenciación alude a la capacidad del discurso periodístico para ofrecer un sistema de referencias, connotaciones y símbolos con el objetivo de interpretar la realidad. Con esto, el discurso pretende orientar la interpretación pública en un sentido preciso: les dice a sus audiencias cómo pensar.

Resulta interesante vincular estas operaciones del discurso periodístico a la comunicación intercultural, ya que no necesariamente todas las temáticas se comportarán de igual manera ni atacarán las mismas áreas discursivas, sobre todo tomando en cuenta que las noticias interculturales presentes en los medios de comunicación tienden a ser minoritarias. Por ejemplo, la valoración hace que las noticias interculturales compitan con secciones informativas más populares, siguiendo la lógica de que lo local, internacional, deportivo y político arrojan más noticias y de aparente mayor interés para las audiencias.

Por su parte, la tematización demuestra que el tratamiento de temas interculturales es, generalmente, negativo y está asociado a conflictos, lo que implica la propagación de estereotipos. Otro ejemplo, la creación de estereotipos negativos frente a la inmigración es útil para los medios, ya que con esto pueden dirigir mensajes más efectivos gracias a la generalización que implica estereotipar.

La actorización demuestra que en los temas interculturales, las culturas minoritarias y marginales tienen una gran visibilidad en los medios, pero son silenciadas en el discurso ante los relatos de actores oficiales.

En definitiva, la referenciación en tópicos interculturales hace que los medios presenten noticias ligadas a conflictos bélicos o a la ilegalidad de los inmigrantes, sobre todo si se trata de tráfico y consumo de drogas.

En resumen, hemos visto cómo los discursos de los medios de comunicación generan construcciones que se traducen en actitudes positivas o negativas hacia otras culturas. Estrella Israel (2006) rescata la existencia de una desigualdad en el tratamiento noticioso de temas interculturales, por lo que propone un modelo para analizar esta diferencia basado en tres dimensiones. En primer lugar, el contexto de producción, donde un grupo de comunicación, un medio y los productores periodísticos aportarán sus sesgos al emitir 
mensajes periodísticos interculturales. En segundo lugar, el texto noticioso como representante de las estrategias discursivas, donde se encuentran tanto líneas editoriales y elementos de connotación como estilos y formatos de representación. En tercer lugar, la comparación con otros discursos presentes en el circuito informativo donde los acontecimientos son procesados y presentados de modo similar o diferente por los medios, lo que faculta la intertextualidad de los mensajes periodísticos.

Gracias a esta base teórica, podemos rescatar la importancia de los niveles textuales y contextuales a la hora de proponer una metodología de análisis de noticias de corte intercultural como lo presentaremos a continuación.

\section{ACD para un análisis de prensa intercultural}

Van Dijk (1990, 1997, 1999, 2003), uno de los principales exponentes del Análisis Crítico del Discurso (ACD), sostiene que los medios de comunicación son el principal instrumento de expresión de los grupos que controlan las mayores cuotas de poder en la sociedad occidental. Por esto, los discursos de los medios de comunicación, sobre todo las noticias, contienen elementos discursivos que refuerzan y reproducen la supremacía de las elites frente a los grupos sociales menos favorecidos. Es decir, los medios de comunicación son los principales productores de discursos sobre desigualdad social. Al respecto, van Dijk ha desarrollado una línea de trabajo por medio del ACD destinada a analizar el modo cómo la prensa escrita presenta a las minorías étnicas y a los inmigrantes.

De acuerdo a lo mencionado y dado que parte de esta investigación pretende comprender cómo los medios de comunicación influyen en la sociedad construyendo imaginarios sociales que refuerzan la diferencia intercultural para con lo peruano, resulta interesante trabajar bajo los lineamientos -y bajo ciertas adaptaciones a la realidad localque propone van Dijk sobre el ACD y sus consecuencias en virtud a lo estipulado en el marco teórico recién expuesto.

En específico, este artículo propone presentar, a modo de ejemplo para un análisis ACD, las noticias referentes a lo peruano en el periódico "El Mercurio", tomando como punto de partida dos noticias seleccionadas y publicadas durante el primer semestre de 2010. Éste análisis se aplicó de lo general a lo particular, de lo global a lo local, en aspectos formales y de significados, y se dividió en cuatro niveles, presentados en dos planos: 


\begin{tabular}{|l|l|}
\hline Plano significado/texto & Nivel temático: significados globales \\
\cline { 2 - 2 } & $\begin{array}{l}\text { Nivel de significados locales: } \\
\text { - De carácter implícito o indirecto } \\
\text { - De carácter explícito o directo }\end{array}$ \\
\hline Plano formal/texto-contexto & Estructuras formales sutiles \\
\cline { 2 - 2 } & Nivel Contextual \\
\hline
\end{tabular}

\subsection{Plano significado/texto}

3.1.1. Nivel temático: significados globales. Se refiere a los temas de los qué trata el discurso periodístico-informativo analizado, marcando las interacciones, la estructura social y las orientaciones de cómo se emite la noticia. En la práctica, estos significados globales, generalmente, no pueden ser reconocidos con tanta evidencia, sino que deben ser inferidos del propio discurso o, en el último de los casos, asignados a él. Con esto, se obtiene una primera idea general de la noticia analizada y se controlan otros aspectos generales de la misma. En el periodismo, este plano de abstracción consiste en un conjunto de categorías textuales convencionales tales como portada, llamados, el texto que conforma la noticia, opiniones y comentarios. Los periodistas, al titular, otorgan la estructura de relevancia del texto, es decir, tratan de esquematizar lo esencial entre el epígrafe y el título de la noticia. Se intenta, en consecuencia, buscar respuesta a varias interrogantes, tales como de qué habla el texto, qué macroestructuras semánticas (temas) es posible deducir del discurso, cómo se unen, cuáles son los presupuestos que se manejan acerca del discurso sobre el otro que ocupa a esta investigación en general.

3.1.2. Nivel de significados locales. Se refiere a un estudio de los significados locales como, por ejemplo y bajo los intereses prioritarios de esta investigación, el significar literal de las palabras. Los significados locales, a diferencias de los significados globales, resultan de la relación que se realiza desde quienes emiten la noticia en función a los modelos mentales y, por lo mismo, en las posteriores opiniones, actitudes y construcciones sociales de quienes reciben esa información. Las formas de significados locales se pueden dividir en dos categorías: de carácter implícito y de carácter explícito.

- De carácter implícito o indirecto, son informaciones que se pueden inferir de palabras del texto, como implicaciones, presupuestos, alusiones, ambigüedades, hipérboles, etc. El texto no lo expresa explícitamente. Este tipo de significado local es parte del modelo mental del público y no está presente con evidencia en el texto analizado. 
- De carácter explícito o directo, son informaciones evidentes que se encuentran con claridad en las palabras del texto. Particularmente, en este paso, interesa indagar acerca de las estrategias léxicas utilizadas por los periodistas, en las informaciones de prensa u noticias sobre las temáticas arrojadas en el nivel anterior.

\subsection{Planoformal/texto-contexto}

3.2.1. Estructuras formales sutiles. Se refiere a las formas o formatos globales y locales que se pueden caracterizar por incidir menos en el control consciente de las noticias por parte de los receptores. Su objetivo es, en principio, observar cómo operan los aparatos ideológicos formales del discurso que pueden emitir falacias, omitir información crucial, construyendo modelos sesgados e interesados de acuerdo a los discursos de dominación y sus fuentes, basado en lo que Potter (1998) defiende como economía de la verdad.

3.2.2. Nivel contextual. Son representaciones mentales de la memoria a largo plazo, donde se almacenan los conocimientos y las opiniones sobre lo vivido. Se refiere a la importancia que tienen en relación con los contextos modélicos locales y globales.

\section{Ejemplos para una aplicación metodológica}

A partir de la aplicación de la antes mencionada herramienta metodológica en algunas noticias publicadas en relación a lo peruano en las páginas del diario "El Mercurio" durante los primeros meses de 2010, podemos ofrecer ciertas lecturas generales que se acercan a las pretensiones de este proyecto de investigación.

El primer paso para el proceso de análisis del material periodístico, objeto de estudio de este trabajo, consistió en seleccionar todas las noticias que hicieran referencia a temas vinculados con este país limítrofe durante lo transcurrido de 2010. Como ya se precisó y para el efecto de este artículo, sólo se presentarán, a modo de ejemplo, dos casos que denoten con la mayor claridad posible la aplicación metodológica a través de la matriz ACD ya desarrollada. Las noticias elegidas y su análisis son las siguientes:

\section{NOTICIA 1 - 27.03.2010}

Estudio de hipotético sismo de 8 grados:

Terremoto en Lima dejaría más de 50 mil muertos

Un sismo de gran magnitud en la capital peruana seguido de un maremoto similar al que azotó a Chile en febrero pasado dejaría más de 50.000 muertos, 600.000 heridos y unas 
200.000 viviendas destruidas, según señala un informe del estatal Instituto Nacional de Defensa Civil.

El documento establece los probables estragos ante un terremoto de 8 grados Richter en la capital peruana y el vecino puerto del Callao, el cual ha sido devastado varias veces por maremotos en el pasado.

El alto índice de muertes se fundamenta en la precariedad de numerosas viviendas de adobe, sobre todo de los barrios antiguos de la zona céntrica de Lima, y por numerosas viviendas sobre los cerros que circundan la capital, que forman barrios marginales, edificadas sin técnicas antisísmicas.

La difusión de este informe coincide con la puesta en marcha ayer de un Plan de Prevención de Sismos 2010, aprobado por el gobierno peruano, tras el impacto del terremoto en Chile. Dicho plan prevé la realización de jornadas de inspección de viviendas en Lima y el Callao para identificar las zonas de acuerdo a su peligrosidad.

\section{Análisis 1}

\begin{tabular}{|c|c|c|c|}
\hline \multirow{2}{*}{\multicolumn{2}{|c|}{$\begin{array}{l}\text { Estudio de hipotético sismo de } 8 \text { grados: } \\
\text { Terremoto en Lima dejaría más de } 50 \text { mil muertos }\end{array}$}} & \multicolumn{2}{|c|}{ El Mercurio } \\
\hline & & \multirow{2}{*}{$\begin{array}{l}\text { Fecha } \\
\text { Página }\end{array}$} & \multirow{2}{*}{$\begin{array}{l}27 / 03 / 2010 \\
\text { A4 }\end{array}$} \\
\hline & & & \\
\hline & & Sección & Internacional \\
\hline \multirow[t]{2}{*}{$\begin{array}{l}\text { Plano } \\
\text { Significado/texto }\end{array}$} & \multicolumn{3}{|c|}{$\begin{array}{l}\text { Nivel temático: significados globales } \\
\text { Esta noticia habla sobre las debilidades del pueblo peruano para } \\
\text { enfrentar un terremoto de las mismas características que el que } \\
\text { azotó a Chile en febrero de } 2010 \text {. }\end{array}$} \\
\hline & \multicolumn{3}{|c|}{$\begin{array}{l}\text { Nivel de significados locales } \\
\text { De carácter implícito: } \\
\text { - "estudio hipotético": Se trata de una comparación hipotética, ya } \\
\text { que el estudio recoge los parámetros del terremoto chileno para } \\
\text { evaluar las condiciones de seguridad en Perú. } \\
\text { - "50 mil muertos": Se trata de una cifra } 6 \text { o } 7 \text { veces más grande que } \\
\text { la del número de muertos en Chile para el terremoto de } 2010 . \\
\text { - "numerosas viviendas de adobe”: Al hablar sobre seguridad ante } \\
\text { un terremoto, las viviendas de adobe se relacionan a una precariedad } \\
\text { en la construcción. Este tipo de viviendas son populares en } \\
\text { Centroamérica por el clima árido subtropical desértico. } \\
\text { - "sin técnicas antisísmicas": Se refiere, indirectamente, al uso de } \\
\text { tecnologías. }\end{array}$} \\
\hline
\end{tabular}




\begin{tabular}{|l|l|}
\hline & $\begin{array}{l}\text { De carácter explícito: } \\
\text { - "barrios marginales": Se refiere a una marginalidad ligada a la } \\
\text { ruralidad, por encontrarse en los cerros, lo que se asocia a barrios } \\
\text { pobres. }\end{array}$ \\
\hline $\begin{array}{l}\text { Plano } \\
\text { Formal/texto- } \\
\text { contexto }\end{array}$ & $\begin{array}{l}\text { Estructuras formales sutiles } \\
\text { Se establece una comparación entre Chile y Perú no comprobable, } \\
\text { donde se valida la preparación chilena por el hecho de tener menos } \\
\text { víctimas que en el hipotético caso peruano. } \\
\text { La realidad que se muestra sobre lo peruano está relacionada con la } \\
\text { falta de seguridad, pobreza, marginalidad y tecnologías precarias. } \\
\text { Por esto, ya que se comparan ambos países, se recurre a una imagen } \\
\text { donde Chile es mejor que Perú y donde este último es un país } \\
\text { inseguro. }\end{array}$ \\
\hline $\begin{array}{l}\text { Nivel contextual } \\
\text { Perú tiene casi el doble de habitantes que Chile, llegando en 2009 a } \\
\text { más de 29 millones. }\end{array}$ \\
\hline
\end{tabular}

\section{NOTICIA 2 - 09.05.2010}

Presidentes de Chile y Perú zanjarán temas y enfoques de relación bilateral:

Piñera agenda primera cita con Alan García y cena con Aznar en Madrid

El ex gobernante español fue uno de los apoyos internacionales durante la campaña presidencial del año pasado. El 11 de marzo pasado, en medio del cambio de mando, sólo se habían saludado. Por lo mismo, el de ahora será el primer diálogo en que Sebastián Piñera y Alan García abordarán con mayor profundidad la compleja relación bilateral.

Ambos conversarán el lunes o martes de la próxima semana en Madrid, cuando participen de la cumbre entre América Latina y la Unión Europea.

La idea, dicen fuentes diplomáticas, es que los presidentes zanjen los temas y el enfoque que conformarán la agenda bilateral, la que había sido congelada por la administración Bachelet tras la demanda limítrofe en La Haya.

Esto ya había sido abordado por los cancilleres Alfredo Moreno y José Antonio García Belaunde el martes pasado, en Buenos Aires, durante la cumbre de Unasur, quienes -tras las acusaciones peruanas de "carrera armamentista"- acordaron retomar la creación de un sistema para homologar la medición del gasto militar.

De hecho, el propio Piñera conversó con el ministro peruano y le dijo que estaba dispuesto a reanudar el mecanismo " $2+2$ ", que reúne a titulares de RR.EE. y Defensa, cuya última cita fue el 2006.

La idea conversada por los cancilleres es dar un fuerte reimpulso a la relación, encapsulando definitivamente en el tribunal de La Haya el tema de la demanda limítrofe interpuesta por Lima. De hecho, en paralelo a la cita con García, Piñera se reunirá el lunes 
con el grupo de abogados extranjeros contratados por el Estado chileno para su defensa ante la corte internacional.

Además de García, Piñera apuesta por sostener reuniones bilaterales con el francés Nicolás Sarkozy y la alemana Angela Merkel. También tendrá un encuentro con el anfitrión, el español José Luis Rodríguez Zapatero.

Pero otra de las citas importantes para el Mandatario se producirá la noche del domingo, cuando se reúna a cenar con el ex presidente del gobierno español José María Aznar.

Ambos son amigos desde hace varios años. Incluso, el ex gobernante ibérico apoyó activamente la campaña presidencial de Piñera, siendo uno de los invitados principales a la ceremonia en septiembre pasado en el Centro de Extensión de la Universidad Católica, donde el entonces abanderado de la Coalición por el Cambio recibió las conclusiones programáticas de los llamados grupos Tantauco.

"Estoy convencido de que será el próximo Presidente de Chile", dijo en esa oportunidad el ex presidente español.

\section{Análisis 2}

\begin{tabular}{|c|c|c|c|}
\hline \multirow{3}{*}{\multicolumn{2}{|c|}{$\begin{array}{l}\text { Presidentes de Chile y Perú zanjarán temas y enfoques de } \\
\text { relación bilateral } \\
\text { Piñera agenda primera cita con Alan García y cena con } \\
\text { Aznar en Madrid }\end{array}$}} & \multicolumn{2}{|c|}{ El Mercurio } \\
\hline & & \multirow{2}{*}{$\begin{array}{l}\text { Fecha } \\
\text { Página }\end{array}$} & \multirow{2}{*}{$\begin{array}{l}\text { 09/05/2010 } \\
\mathrm{C} 2\end{array}$} \\
\hline & & & \\
\hline & & Sección & Política \\
\hline \multirow[t]{2}{*}{$\begin{array}{l}\text { Plano } \\
\text { Significado/texto }\end{array}$} & \multicolumn{3}{|c|}{$\begin{array}{l}\text { Nivel temático: significados globales } \\
\text { Esta noticia expone las principales actividades de la agenda semanal } \\
\text { de Sebastián Piñera. Si bien, a nivel de significados globales, no existe } \\
\text { relación entre la reunión de Piñera con García y su visita a Aznar, se } \\
\text { trata de actividades del área internacional. Por lo tanto, las relaciones } \\
\text { con Perú son prioritarias para la agenda internacional del presidente } \\
\text { Piñera. }\end{array}$} \\
\hline & \multicolumn{3}{|c|}{$\begin{array}{l}\text { Nivel de significados locales } \\
\text { De carácter implícito: } \\
\text { - "zanjarán": hace referencia a una solución definitiva al conflicto } \\
\text { entre países, pero no puede ser definitiva al tener temas judiciales y } \\
\text { políticos pendientes, como la demanda ante La Haya, por lo que, por } \\
\text { un lado, esta noticia expone la iniciativa de ambos gobiernos por } \\
\text { conformar una agenda bilateral, es decir, una solución a largo plazo, y, } \\
\text { por otro lado, apresura la necesidad de terminar con las acciones } \\
\text { legales ante la mirada de un tercero: Europa y las relaciones } \\
\text { económicas con ese continente. }\end{array}$} \\
\hline
\end{tabular}




\begin{tabular}{|c|c|}
\hline & $\begin{array}{l}\text { - "relación bilateral”: Se refiere, indirectamente, a los conflictos entre } \\
\text { Chile y Perú, ya que no se exponen otro tipo de relaciones que no sean } \\
\text { sobre carreras armamentistas o juicios pendientes. } \\
\text { - "sistema para homologar la medición del gasto militar": Se trata } \\
\text { de una medida en contra de una carrera armamentista. } \\
\text { - "en paralelo a la cita con García, Piñera se reunirá el lunes con } \\
\text { el grupo de abogados extranjeros contratados por el Estado } \\
\text { chileno para su defensa ante la corte internacional”: Quiere } \\
\text { decir que la reunión con el presidente peruano corresponde a un } \\
\text { acto de camaradería, sin la posibilidad de resolución ante los } \\
\text { conflictos actuales. Se muestra un doble juego; por un lado, los } \\
\text { presidentes se reúnen en el marco de un ejercicio de relaciones } \\
\text { exteriores, pero, por otro, continúan las estrategias para sobrellevar } \\
\text { la demanda a La Haya. } \\
\text { De carácter explícito: } \\
\text {-compleja relación bilateral": A pesar de que históricamente } \\
\text { existen conflictos entre Chile y Perú, la complejidad referida está dada } \\
\text { por las acusaciones de carreras armamentistas y por la denuncia ante } \\
\text { La Haya. }\end{array}$ \\
\hline \multirow[t]{2}{*}{$\begin{array}{l}\text { Plano } \\
\text { Formal/texto- } \\
\text { contexto }\end{array}$} & $\begin{array}{l}\text { Estructuras formales sutiles } \\
\text { Se muestran las relaciones bilaterales entre Chile y Perú } \\
\text { exclusivamente como conflictos, basados en las carreras } \\
\text { armamentistas y en la demanda pendiente ante Chile por la cual } \\
\text { Perú pretende modificar sus límites marítimos. } \\
\text { Además, se vinculan las relaciones exteriores de Chile con dos } \\
\text { bandos: por un lado, cuenta con el apoyo de líderes europeos y, por } \\
\text { otro, los conflictos fronterizos vecinales. Por lo tanto, se pretende } \\
\text { establecer una conexión donde las relaciones con Europa son } \\
\text { mejores que las relaciones con los países vecinos. }\end{array}$ \\
\hline & $\begin{array}{l}\text { Nivel contextual } \\
\text { Con respecto a las carreras armamentistas, en los últimos cinco años, } \\
\text { las compras de armamento militar por parte de Chile que han } \\
\text { causado más polémicas en Perú son los helicópteros de transporte } \\
\text { rusos Mil Mi 17, los aviones cazabombarderos F-16 y los tanques } \\
\text { Leopard } 2 \mathrm{~A} 4 \text {. En el primer trimestre del gobierno de Piñera, Perú ha } \\
\text { hecho noticia por mantener negocios de armas con Rusia, China y } \\
\text { Estados Unidos. }\end{array}$ \\
\hline
\end{tabular}




\begin{tabular}{|l|l|}
\hline Según las cifras del Instituto de Estudios para la Paz de Estocolmo \\
(SIPRI), entre 2005 y 2009 la inversión militar en Sudamérica \\
aumentó en un 150\%. El volumen de importaciones de toda \\
América, incluido Estados Unidos, respecto del número global se \\
cifró en un 11\%, el mismo porcentaje registrado durante el período \\
entre 2000 y 2004. En América del Sur, el principal importador de \\
este tipo de armas fue Chile, que ocupa el puesto 13 a nivel mundial. \\
Sobre los tipos de relaciones bilaterales, durante el primer trimestre \\
del gobierno de Sebastián Piñera, las relaciones económicas con Perú \\
han aumentado, sobre todo en lo que respecta a la inversión \\
inmobiliaria. Chile suma 8.430 millones de dólares de inversiones en \\
Perú en los últimos diez años.
\end{tabular}

\section{A modo de conclusión: mirada aproximativa}

En primer lugar, al encontrar una cantidad significativa de construcciones sociales de la realidad ya instauradas en nuestra memoria que hacen referencia a una diferenciación entre un nosotros y lo peruano, podemos deducir que la inserción de noticias en "El Mercurio" que aluden a tales relaciones interculturales son presentadas -como se puede precisar en los ejemplos- como un conflicto, por lo que existe una tensión constante al representar tales problemas desde una postura defensiva y diferenciadora de elementos identitarios.

La consecuencia de esta posición, en términos generales y por parte de los medios de comunicación, hace que las noticias relacionadas con estos temas traten de instaurar modelos de representación en contra del cruce intercultural. Se aprecia que esta generalización se muestra fundamentada, en gran parte de los casos, por la idea de que existe una voz oficial que agrupa a su respectiva nación y que actúa bajo tal generalización para resguardar el bien común y perpetuar el estatus quo. El problema visible de estas generalizaciones es que se puede apreciar que de ella nacen las representaciones sociales o imaginarios que causan discriminación con las otras culturas; el estereotipo, muy unido a la generalización por su idea de agrupación, marca tanto el trato discriminatorio que se le da a los elementos culturales de, en este caso, lo peruano en la prensa estudiada, como las representaciones que pretende instaurar a partir de ella y los nuevos conflictos de encuentro.

Otra generalización a modo de conclusión y sobre el análisis de estas noticias, indica que los medios de comunicación manipulan constantemente los rasgos más delicados de las culturas otras como la idiosincrasia, la estabilidad país, las relaciones bilaterales, 
presentando así las diferencias identitarias desde una perspectiva valórica marcada por una relación de superioridad y sometimiento. Por ejemplo, el caso de la hipotética y poco objetiva cantidad de muertes en un posible terremoto grado ocho en Perú.

Se puede apreciar, sobre todo gracias a los niveles contextuales de este análisis previo y prematuro, que la prensa chilena estudiada carece de rigurosidad periodística. Los temas bilaterales son presentados de manera superficial y utilizando sólo fuentes oficiales y/o hipotéticas, lo que contribuye a que las audiencias creen imaginarios errados sobre las culturas minoritarias que se interrelacionan con lo chileno.

Finalmente y a modo de mirada aproximativa a partir de estos primeros análisis ${ }^{3}$, se podría anunciar que:

1. Las representaciones sociales construidas y confirmadas por el medio de comunicación estudiado promueve estereotipos sobre las diferencias que marcan una distancia con lo chileno. Es decir, existe una tendencia clara, por parte de los medios, de promover lo negativo de otras culturas, lo que se presenta en forma de conflicto.

2. Se justifica el estudio de la comunicación y el periodismo intercultural demostrando que la representación que hacen los medios de comunicación sobre las culturas estudiadas incide fuertemente en los imaginarios que los chilenos hacemos de ellas, lo que demanda una preocupación por proponer espacios de interacción intercultural tanto en los medios como en otras instituciones sociales. En este sentido, el análisis crítico de los medios de comunicación abre un espacio importante para plantear estrategias de interacción cultural a través de diálogos e intercambios entre los distintos actores que conviven en una sociedad.

\section{Referencias bibliográfícas}

Barata, F. (2006): "Inmigración y criminalización en los medios de comunicación”. En Flujos migratorios y su (des) control. Barcelona: Anthropos.

Browne, R.; Baessolo, R. y Silva, V. (2010): "Periodismo intercultural: representación peruana y boliviana en la prensa chilena”. En Comunicar, $n^{\circ}$ 35: Huelva.

Berger, P.y Luckmann, T. (1972): La construcción social de la realidad. Buenos Aires: Amorrortu. Grimson, A. (2001): Interculturalidad y Comunicación. Buenos Aires: Norma.

Israel, E. (2006): Comunic@ción y Periodismo en una Sociedad Global. México: Trillas.

\footnotetext{
${ }^{3}$ Este proyecto, de todas maneras, surge como consecuencia de un trabajo anterior que durante dos años estudió la representación peruana, boliviana y mapuche en la prensa nacional. Fondecyt $\mathrm{n}^{\circ} 11070062$ "Comunicación intercultural y periodismo intercultural: análisis crítico de la construcción social de la realidad a través de la representación mapuche y peruano-boliviana en las noticias de la prensa diaria de cobertura nacional (Copesa y El Mercurio)".
} 
Martínez Nicolás, M. (1996): El estudio del discurso periodístico informativo: Una aproximación metodológica desde la teoría de la discursividad social. Barcelona: Trípodos.

Póo, X. (2009): "Imaginarios sobre inmigración peruana en la prensa escrita chilena: una mirada a la instalación de la agenda de la diferencia”. En Revista F@ro, n9: Valparaíso.

Potter, J. (1998): La representación de la realidad. Discurso, retórica y construcción social. Barcelona: Paidós.

Rodrigo Alsina, M. (1999): Comunicación Intercultural. Barcelona: Anthropos.

Van Dijk, T. (1990): La noticia como discurso. Comprensión, estructura y producción de la información. Barcelona: Paidós.

-- (1997): Racismo y Análisis Crítico de los Medios. Barcelona: Paidós.

(1999): Ideología. Barcelona: Gedisa.

(2003): "La multidisciplinariedad del análisis crítico del discurso: un alegato a favor de la diversidad” en Wodak, R. y Meyer, M.: Métodos de análisis crítico del discurso. Barcelona: Gedisa, pp. 143-178. 